\title{
AMYOTROPHIC LATERAL SCLEROSIS PLUS SYNDROME: A RARE CASE REPORT
}

Vanitha S1, Balaraj K. P2

\section{HOW TO CITE THIS ARTICLE:}

Vanitha S, Balaraj K. P. “Amyotrophic Lateral Sclerosis plus Syndrome: A Rare Case Report”. Journal of Evolution of Medical and Dental Sciences 2015; Vol. 4, Issue 17, February 26; Page: 3012-3016,

DOI: $10.14260 /$ jemds/2015/437

ABSTRACT: Amyotrophic lateral sclerosis (ALS) is a neurodegenerative disorder. In 1994, the El Escorial criteria were proposed for the diagnosis of ALS. These criteria include ALS-plus syndromes, which are defined by an association of ALS with extrapyramidal features or dementia. we present a case report of 31 years young man presented with weakness of lower limbs since one and half years and slurring of speech since 8 months and on examination patient had scanning of speech, eye saccades were slow, upper motor neuron type of quadriparesis with cerebellar ataxia and dysmetria with no sensory or bowel bladder involvement. Routine blood investigations were normal and MRI Brain showed features of atrophy of cerebrum and cerebellum and ENMG shows neurogenic pattern of fasciculations and spontaneous activity. Clinically cerebellar ataxia and dysmetria with pure motor neuron disease suggests the possibility if ALS Plus syndrome especially ubiquitinated forms of TDP43 and ubiquitinated p62-positive inclusions were frequently observed. As such it is the rare presentation in young adults, further studies over ALS plus syndrome will bring additional information about neuropathological details and its effect on outcome.

KEYWORDS: Amyotropic lateral sclerosis, Quadriparesis, Escorial criteria, fasciculations and ubiquinated inclusions.

INTRODUCTION: Amyotrophic lateral sclerosis (ALS) is a progressive neurodegenerative disease that affects the upper and the lower motor neurons in the cerebral cortex, brainstem, and spinal cord. ${ }^{1}$ The clinical features can be considered in relation to neurological regions or levels. Physical signs of this disorder encompass both, the upper and the lower motor neuron findings. Atrophy and weakness of respiratory muscles eventually lead to respiratory failure and death. Amyotrophic lateral sclerosis (ALS)-Plus syndromes meet clinical criteria for ALS but also include 1 or more additional features such as dementia, geographic clustering, extrapyramidal signs, objective sensory loss, autonomic dysfunction, cerebellar degeneration, or ocular motility disturbance. According to the revised El Escorial criteria, the described unusual combination of upper and lower motor neuron signs in association with cerebellar ataxia with dysmetria can be classified as a specific form of ALSplus syndrome..$^{2,3}$

CASE REPORT: We are presenting a case of 31-year-old man who had weakness of limbs for $1 \frac{1 / 2}{2}$ years which was asymmetrical in onset, started in right lower limb ,after 6 months progressed to upper limbs ,6 months later progressed to left lower limb. Patient also presented with Slurring of speech since 8 months. No history of fasciculations, radicular pain, sensory symptoms, bladder and bowel involvement. Family history is not contributing.

NEUROLOGIC EXAMINATION: The patient was awake, alert, and had dysarthria-scanning speech and could appropriately respond to questions and follow commands. His writing was illegible. Pupils 


\section{CASE REPORT}

were 2 to $3 \mathrm{~mm}$ in diameter and reactive to light and near vision. Horizontal and vertical saccades were slow. The uvula was midline and Gag reflex was normal .The sternocleidomastoid muscles and tongue was normal, no atrophy or fasciculations. Rapid tongue movements were normal. Tone was rigid in all the limbs. Power was Medical Research Council (MRC) grade 5 in the deltoid, biceps, and triceps muscles but MRC grade 4 in the right and left wrist flexors and extensors and intrinsic muscles of the hand. There was atrophy of the forearm and hand intrinsic muscles. Leg power was MRC grade 4, he was able to rise from a chair without using his hands, and heel and toe walking was difficult. No fasciculations were noted. Reflexes were exaggerated in the arms with bilateral Hoffman sign. Pendular knee jerk was present with well sustained clonus. Cutaneous abdominal reflexes were present. Babinski sign was positive. Palmomental reflex was present. Sensory examination found to be normal. Cerebellar ataxia with dysmetria was present.

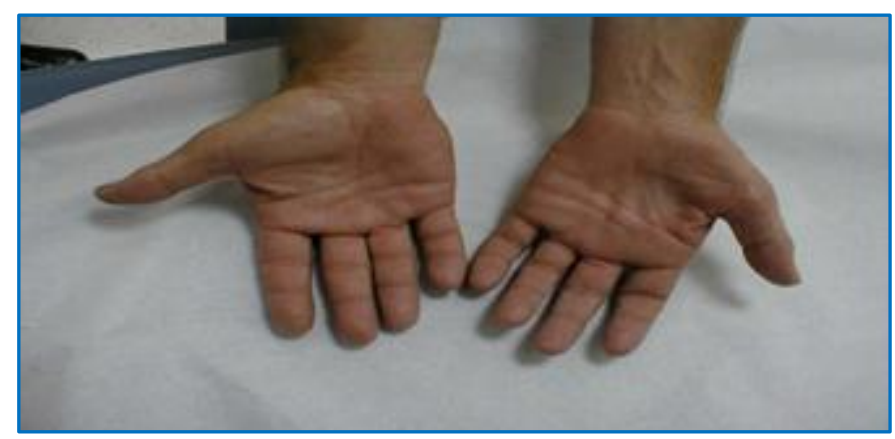

Fig. 1: Showing wasting of

Intrinsic muscles of both hands

Clinical and Laboratory Investigations: Magnetic resonance images of the brain demonstrated diffuse cerebral and cerebellar atrophy changes. An electromyogram demonstrated ongoing chronic partial denervation and fasciculations in muscles of the arm and leg (Biceps \& vastus lateralis). Complete blood cell count, thyroid function studies, serum parathyroid hormone and vitamin B12, electrolytes values found to be normal and HIV-negative.
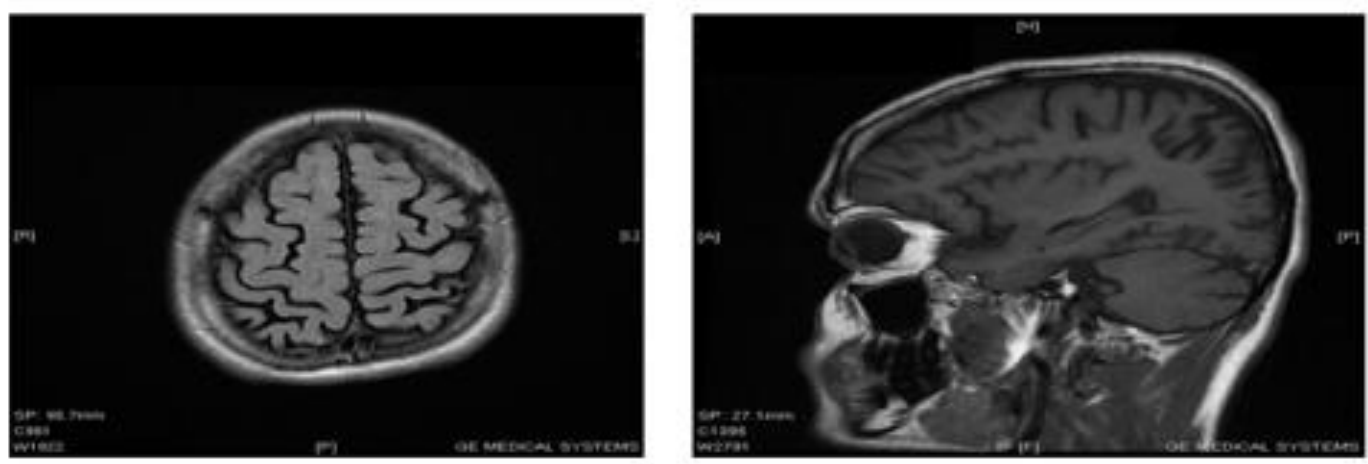

Fig. 2: MRI of brain axial T2 image showing diffuse cerebral atrophy 


\section{CASE REPORT}

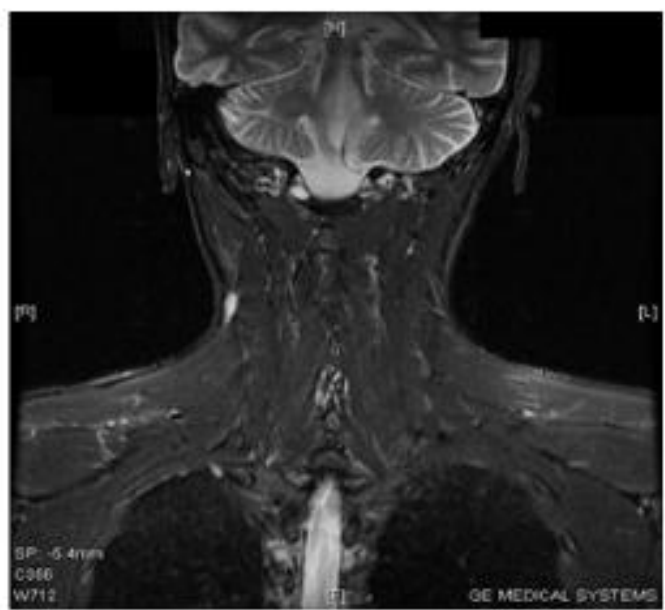

Fig. 3: MRI of brain and c-spine coronal stir image showing diffuse cerebellar atrophy

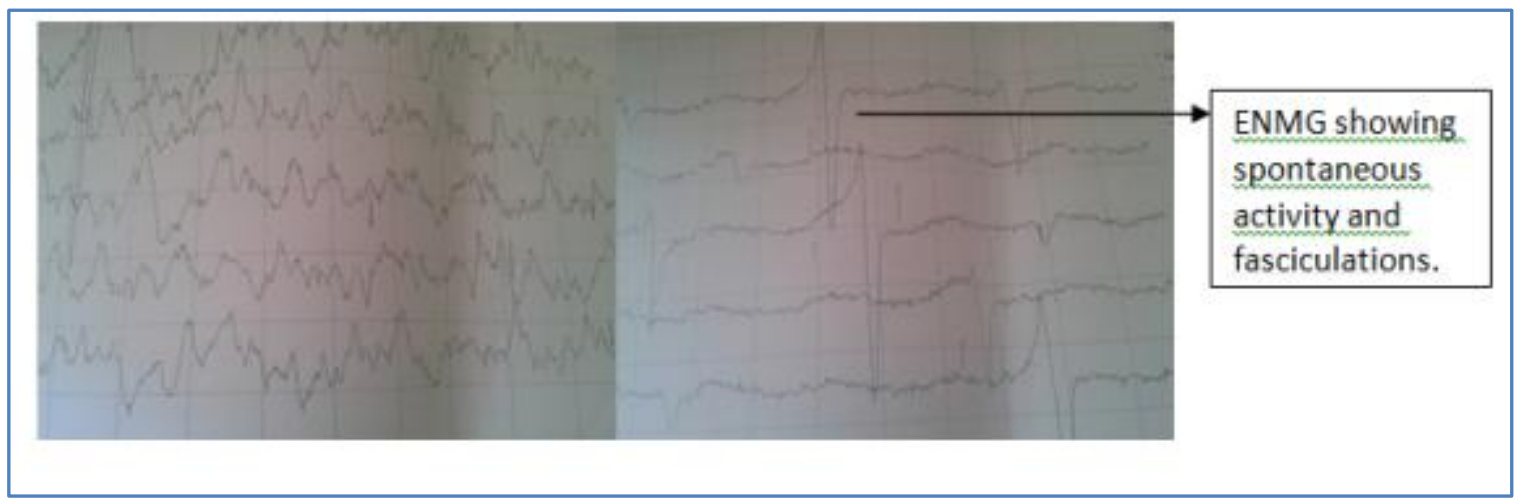

Fig. 4: ENMG showing spontaneous activity and fasciculations which are neurogenic pattern

The patient was given Tab. Riluzole $50 \mathrm{mg}$ twice a day therapy and advised follow up.

DISCUSSION: ALS is a fatal neurodegenerative disease. Patients with ALS become progressively paralyzed, while remaining fully alert mentally, due to degeneration of the upper and the lower motor neurons in the brain and spinal cord. Brain stem involvement produces dysarthria (Slurred speech), dysphagia, and aspiration .Our patient had presented with features of pure motor system involvement affecting upper and lower motor neurons involving limb musculature and cerebellar ataxia.4,5 Electromyography, MRI images and nerve conduction further confirm the diagnosis .According to revised E1 Escorial criteria the combination of upper and lower motor neuron disease with cerebellar ataxia fits specific form of ALS plus syndrome.Literature review showed very few cases of ALS plus syndrome involving cerebellum . Herein, imaging and neuropathological evidence for involvement of the cerebellum, which to date is not thought to be involved in ALS, is reviewed

Evidence for involvement of the cerebellum in ALS comes from several neuropathological studies. Especially ubiquitinated forms of TDP-43 and ubiquitinated p62-positive inclusions were frequently observed. 


\section{CASE REPORT}

The widely used transgenic SOD1-G93A ALS mice model showed prominent cerebellar immunostaining of pERK and alterations of tau expression.6,7 Studies using advanced MRI techniques demonstrated that several cerebral areas, including the cerebellum, were recruited in order to compensate for functional motor decline. Functional MRI, voxel based morphometry, and diffusiontensor imaging showed these cerebellar alterations as being of functional and structural nature.

CONCLUSION In summary, we presented an interesting case of a patient with ALS Plus syndrome involving cerebellum which is rare association. After review of the available literature, it was felt that these were chance occurrences and that treatment entities do not affect the course of progressive ALS/MND. ${ }^{8,9}$ Clinically cerebellar ataxia with pure motor neuron disase suggests the possibility if ALS Plus syndrome especially ubiquitinated forms of TDP-43 and ubiquitinated p62-positive inclusions were frequently observed. As such it is the rare presentation in young adults, further studies over ALS plus syndrome will bring additional information about neuropathological details and its effect on outcome..$^{10}$

\section{REFERENCES:}

1. Nervenarzt 2002 Aug; 73(8):751-3.Cerebellar symptoms in motor neuron diseases. Special form of amyotrophic lateral sclerosis plus syndrome. Schimke N, Krampfl K, Petri S, Dengler R, Bufler J.

2. Brooks BR (1994) El Escorial World Federation of Neurology criteria for the diagnosis of amyotrophic lateral sclerosis. Subcommittee on Motor Neuron Diseases/Amyotrophic Lateral Sclerosis of the World Ferderation of Neurology Research Group on Neuromuscular Diseases and the El Escorial Clinical limits of amyotrophic Lateral sclerosis workshop contributors. J Neurol Sci 124 [Suppl]: 96-107.

3. Brooks BR, Miller RG, Swash M,Munsat TL, World Federation of Neurology Research Group on Motor Neuron Diseases (2000) El Escorial revised: revised criteria for the diagnosis of amyotrophic lateral sclerosis. Amyotroph Lateral Sclerosis and Other Motor Neuron Disorders 1 (5): 293-9.

4. Horiuchi I, Furuya H, Yoshimura T, Kobayashi S (1997) A case of severe involvement of the motor neuron system accompanied with cerebellar ataxia. Clin Neurol 37: 123-126.

5. Murakami N, Yoshida M,Hashizume Y, Muroga T, Takahashi (1989) A nosological study of a patient showing ataxia and lower motor neuron involvement. Clin Neurol 29: 1116-1121.

6. Machida Y, Tsuchiya K, Anno M, Haga C et al. (1999) Sporadic amyotrophic lateral sclerosis with multiple system degeneration: a report of an autopsy case without respirator administration. Acta Neuropathol (Berl) 98(5): 512-5.

7. The involvement of the cerebellum in amyotrophic lateral sclerosis Prell T, Grosskreutz J Amyotrophic Lateral Sclerosis Fronto temporal Degeneration. 2013 Dec; 14(7-8):507-15. doi: 10.3109/21678421.2013.812661. Epub 2013 Jul 29.

8. Koerner DR (1952) Amyotrophic lateral sclerosis on Guam. A clinical study and review of the literature. Ann Intern Med 37: 1204-1220.

9. Gajdusek DC, Salazar AM (1982) Amyotrophic lateral sclerosis and Parkinsonian syndroms in high incidence among the Auya and Jakai people of West New Guinea. Neurology 32: 107-126. 


\section{CASE REPORT}

10. Cerebellar and frontal lobe involvement in ALS as determined by MR DTI AM Ulug, R Watts, AP Hays - Proceedings of the 13th , 2005 - cds.ismrm.org.

\section{AUTHORS:}

1. Vanitha S.

2. Balaraj K. P.

\section{PARTICULARS OF CONTRIBUTORS:}

1. Post Graduate, Department of General Medicine, Kempegowda Institute of Medical Sciences, Bangalore.

2. Professor, Department of General Medicine, Kempegowda Institute of Medical Sciences, Bangalore.

\section{FINANCIAL OR OTHER} COMPETING INTERESTS: None

\section{NAME ADDRESS EMAIL ID OF THE CORRESPONDING AUTHOR:}

Dr. Vanitha S, Room No -407, KIMS Resident Quarters, KIMS Hospital Campus, V. V. Puram, Bangalore-560004.

E-mail: drvani85@gmail.com

Date of Submission: 02/02/2015.

Date of Peer Review: 03/02/2015.

Date of Acceptance: 17/02/2015.

Date of Publishing: 26/02/2015. 